\title{
Some positive results in the context of universal models
}

\author{
Mirna Džamonja
}

\begin{abstract}
Let $(\mathcal{K}, \leq)$ be a quasi-ordered set or a class, which we think of as a class of models. A universal family in $\mathcal{K}$ is a dominating family in $(\mathcal{K}, \leq)$, and if there is such a family of size one then we call its single element a universal model in $\mathcal{K}$. We survey some important instances of the existence of small universal families and universal models in various classes and point out the influence of the axioms of set theory on the existence of such objects. Then we present some of the known methods of constructing small universal families and universal models and discuss their limitations, pointing out some of the remaining open questions.
\end{abstract}

\section{Introduction}

Let $(\mathcal{K}, \leq)$ be a a quasi-ordered set or a class, which we think of as a class of models. In the context that interests us this may be the class of models of a given cardinality of some first order theory ordered by elementary embedding or the class of models of a given cardinality of some abstract elementary class quasi-ordered by the inherited order. We may also consider classes whose membership is not determined by cardinality but by some other cardinal invariant such as topological weight. A universal family in $\mathcal{K}$ is a dominating family in $(\mathcal{K}, \leq)$, and if there is such a family of size one then we call its single element a universal model in $\mathcal{K}$. The smallest size of a universal family is called the universality number of $(\mathcal{K}, \leq)$.

Immediate examples of universal models are the the rationals considered as a linear order, which embed every countable linear order, or $[0,1]^{\kappa}$ which contains a closed copy of every compact space of weight $\kappa$, or the random graph which embeds every countable graph. There are many other examples in just about every branch of mathematics. The purpose of this article is to discuss general methods which can be used to demonstrate the existence of universal models in various specific contexts. In this presentation we concentrate on countable first order theories. The article does not deal with the related subject of methods that can be used to demonstrate that a certain theory does not have a small universal family at a certain cardinal; we can refer the reader to the survey article $[\mathbf{2}]$ for a description of some such ideas.

1991 Mathematics Subject Classification. Primary 03E35, 03 C55.

Key words and phrases. universal models, universal families.

The author thanks Mittag-Leffler Institute for their support in September 2009 and EPSRC for their support through grant EP/G068720. 
The article is organised as follows: it first presents some classical results from model theory that apply in $Z F C$ or under the $G C H$-like assumptions. This is the content of $\S 2$, which is divided to subsections relating to saturated and special models and countable universal models. Section 3 moves into the realm where $\mathrm{CH}$ is violated and considers the possible existence of universal models in forcing extensions where $C H$ fails, concentrating on $\aleph_{1}$. This section is divided into subsections dealing with graphs, triangle-free graphs, linear orders and amenability at $\aleph_{1}$.

Throughout $\kappa$ stands for an infinite cardinal. An unattributed $T$ stands for a theory, which means a complete first order theory with infinite models. For simplicity in this presentation we restrict ourselves to the case of countable theories. A type for us is any consistent set of sentences, and a complete type is a maximal consistent set of sentences. By a universal model of $T$ of size $\kappa$ we mean a model in which every other model of $T$ of size $\kappa$ embeds elementarily ${ }^{1}$.

\section{Some classical results}

We present some results on the existence of certain kinds of universal models for complete first order theories, again restricting to the case of countable theories. Results presented in this section mostly come from 1960s.

\subsection{Saturated and special models.}

Definition 2.1. A model $M$ of a theory $T$ is said to be $\kappa$-saturated if for every $A \subseteq M$ of size $<\kappa$, the expansion $(M, a)_{a \in A}$ realises every type $\Gamma(x)$ of the expanded language which is consistent with the complete theory $\operatorname{Th}(M, a)_{a \in A} . M$ is said to be saturated if it is $|M|$-saturated.

A generalisation of Cantor's proof that the rationals are a unique countable dense linear order with no first or last elements, gives us that saturated models are universal. See Theorem 2.4 for a detailed statement. The basic theorem about the existence of saturated models at uncountable cardinals is the following

Lemma 2.2. (Vaught, [10]) Suppose that $N$ is a model $T$ of size $\leq 2^{\kappa}$. Then there is a $\kappa^{+}$-saturated extension $M$ of $N$ of size $2^{\kappa}$.

Proof. Note that $\left|[N]^{\kappa}\right|=2^{\kappa}$, and for every $A \in[N]^{\kappa}$ the language $\mathcal{L}^{A}=$ $\mathcal{L} \cup\left\{c_{a}\right\}_{a \in A}$ has size $\kappa$, therefore the total number of relevant types is $2^{\kappa}$. Introduce for each such type $\Sigma$ a new symbol $c_{\Sigma}$. We can form the set of sentences $\Gamma$ consisting of the elementary diagram of $N$ along with $\Sigma\left(c_{\Sigma}\right)$ for all relevant $\Sigma$. This is a finitely satisfiable set of sentences, hence it has a model, so it has a model of size $2^{\kappa}$. Let $M$ be the reduction of this model to the original language. $\star_{2.2}$

Provided that we assume some cardinal arithmetic this now gives us the existence of saturated models in successor cardinals:

THEOREM 2.3. (Vaught, [10]) Suppose that $\kappa$ satisfies $2^{\kappa}=\kappa^{+}$. Then there is a saturated model $M$ of $T$ of size $\kappa^{+}$.

Proof. Let $N$ be any model of $T$ of size $2^{\kappa}$. By induction on $\alpha<2^{\kappa}$ we choose models $N_{\alpha}$ of $T$ so that

- $N_{0}=N, N_{\alpha} \prec N_{\alpha+1}, N_{\delta}=\bigcup_{\alpha<\delta} N_{\alpha}$ for $\delta$ limit $>0$,

\footnotetext{
${ }^{1}$ In this context, because of the compactness theorem, we could equivalently require that every model of infinite size $\leq \kappa$ embeds into the $\kappa$-universal model.
} 
- For every $A \subseteq N_{\alpha}$ of size $\kappa$ and type $\Gamma(x)$ consistent with the complete theory of $\left(N_{\alpha}, a\right)_{a \in A}, \Gamma(x)$ is realised in $N_{\alpha+1}$.

To do the construction at the successor stage $\alpha+1$ we simply apply Lemma 2.2 to the model $N_{\alpha}$. At the end let $M=\bigcup_{\alpha<\kappa^{+}} N_{\alpha} \cdot \star_{2.3}$

Saturation is not necessary for universality. A weaker notion that still suffices is that of a special model: a model $M$ of size $\kappa$ is a special model if it is the union of an elementary chain $\left\langle M_{\lambda}: \lambda\left\langle\lambda^{*}\right\rangle\right.$ such that each $\lambda$ is a cardinal and $M_{\lambda}$ is $\lambda^{+}$-saturated. By definition, saturated models are special. The opposite is not true. Relationship between saturation, speciality and universality is given by the following:

THEOREM 2.4. Every saturated model is special and every special model is universal.

Proof. The first sentence follows by definition. Suppose now that $M$ is the union of a specializing chain $\left\langle M_{i}: i\left\langle i^{*}\right\rangle\right.$ where $M_{i+1}$ is $\kappa_{i}$-saturated for some cardinals $\kappa_{i}$ increasing to $\kappa$, which is the size of $M$. We may without loss of generality assume that this chain is continuous. Let $N$ be a model of $T$ of size $\kappa$, enumerated as $\left\{x_{\alpha}: \alpha<\kappa\right\}$. By induction on $\alpha$ we choose $y_{\alpha} \in M$ such that $x_{\alpha} \mapsto y_{\alpha}$ is an elementary embedding. We choose $y_{\alpha} \mathrm{s}$ in blocks of $\kappa_{i}$ for $i<i^{*}$, that is the induction is on $i$, so that $\alpha<\kappa_{i} \Longrightarrow y_{\alpha} \in M_{i}$.

Suppose that $\left\langle y_{\alpha}: \alpha<\kappa_{i}\right\rangle$ have been chosen. Choose $y_{\alpha} \in M_{i+1}$ for $\alpha \in$ $\left[\kappa_{i}, \kappa_{i+1}\right)$ by induction on $\alpha$. Suppose that $\alpha<\kappa_{i+1}$ and $y_{\beta}$ for $\beta<\alpha$ have been chosen. We use a modification of Cantor's idea from the proof of the uniqueness of the rationals: let $\Gamma(x)$ be the type of $x_{\alpha}$ in $\left(N, x_{\beta}\right)_{\beta<\alpha}$. Therefore $\Gamma$ is a type of size $<\kappa_{i+1}$ in $\left(M_{i+1}, y_{\beta}\right)_{\beta<\alpha}$. By the saturation of $M_{i+1}$ we can find $y_{\alpha} \in M_{i+1}$ which realises this type and the induction continues. $\star_{2.4}$

TheOREM 2.5. (Morley and Vaught, $[\mathbf{1 0}]$ ) Suppose that $\kappa=2^{<\kappa}$ is uncount$a b l e^{2}$. Then there is a special model of $T$ of size $\kappa$.

Proof. If $\kappa=\lambda^{+}$then by the assumption $2^{\lambda}=\kappa$ and hence by Theorem 2.3 there is a saturated model of $T$ of size $\kappa$. Suppose then that $\kappa$ is a limit cardinal. Our assumptions allow us to choose an increasing sequence $\left\langle\kappa_{i}: i\left\langle i^{*}\right\rangle\right.$ of infinite cardinals with limit $\kappa$, and such that $2^{\kappa_{i}}=\kappa_{i+1}$. Then we build an elementary chain $\left\langle M_{i}: i<i^{*}\right\rangle$ by starting with any model $M_{0}$ of $T$ of size $\kappa_{0}$, and applying Lemma 2.2 at successor stages to get a model $M_{i+1}$ of size $\kappa_{i+1}$ which is $\kappa_{i}{ }^{+}$-saturated. Letting $M=\bigcup_{i<i^{*}} M_{i}$ we obtain a special model as required. $\star_{2.5}$

CONCLUSION 2.6. Every countable first order theory $T$ has a universal model of size $\kappa$ for every $\kappa>\aleph_{0}$ satisfying $2^{<\kappa}=\kappa$.

Results presented above can be found in Chapter V of [1]. We also quote a selection of theorems which show that some assumptions on the kind of theories and on cardinal arithmetic are necessary for this conclusion.

THEOREM 2.7. (1) (Hausdorff, [5]) There exists a saturated linear order of size $\kappa>\aleph_{0}$ iff $\kappa=\kappa^{<\kappa}$.

(2) (Shelah, see [7] for a proof) Suppose that $V$ is a model of $G C H$ in which $\kappa$ is a regular cardinal, and let $G$ be $V$-generic for the Cohen forcing which adds

\footnotetext{
${ }^{2}$ The role of uncountability here is that we need $\kappa$ to be larger than the size of $T$.
} 
$\kappa^{++}$Cohen subsets to $V$. Then in $V[G]$, in power $\kappa^{+}$, there is no universal graph, linear order, or generally model of a complete first order $T$ which is unstable in $\kappa$.

(3) (Kojman-Shelah, [7]) Suppose $\kappa$ is a regular cardinal and there is some cardinal $\lambda$ such that $\lambda^{+}<\kappa<2^{\lambda}$. Then there is no universal linear order of cardinality $\kappa$.

Note that if $T$ is stable in $\kappa$ then it follows by an argument analogous to that of Theorem 2.8 below that $T$ has a saturated model of size $\kappa$.

2.2. Countable universal models. Countable universal models are abundant in mathematics. For example, in addition to the examples we mentioned in the Introduction there are examples in topology: any countable dense subset of the Urysohn space is countably universal in the class of metric spaces with almost isometric embedding. In model theory however, the theory of countable models is different from the theory of the uncountable ones - as is most strikingly witnessed by the well developed classification theory using the number of pairwise non-isomorphic models [11], versus the still unresolved Vaught conjecture about countable models. In the theory of universal models it is similarly the case that countable models have a special role. Specifically, the methods presented in the rest of this paper tend to apply only to uncountable models. However, this is not the case with the concept of saturated models, which makes perfect sense in the case of $\kappa=\aleph_{0}$, see Definition 2.1. In fact, the concept of saturation was first introduced in the countable case, by Vaught. Exactly the same proof as in the uncountable case applies to show that saturated models are universal. For example, a well known example of a countable universal model is the linear order of the rationals, and the rationals are an example of a saturated model. In the case of countable models there is a syntactic characterisation of the existence of saturated models, due to Vaught in [17]. It also appears as Theorem 2.3.7 in [1].

THEOREM 2.8. (Vaught, [17]) T has a countable saturated model iff for every $n<\omega, T$ has only countably many complete types in $n$-variables.

PROOF. In the forward direction, let $M$ be a countable saturated model of $T$. Then for every $n$ and complete type $\Gamma$ in $n$ variables, $\Gamma$ is realised by an $n$-tuple in $M$. Since there are only countably many such tuples and none of them can realise more than one complete type, the conclusion follows.

In the other direction, we shall expand $T$ to a maximal consistent theory $T^{*}$ in an expanded language, such that any countable model of $T^{*}$ gives us a saturated model of $T$. Let us form the language $\mathcal{L}^{*}$ by adding countably many new constant symbols $\left\{c_{n}: n<\omega\right\}$ to $\mathcal{L}$. Let $\left\{\varphi_{n}: n<\omega\right\}$ be an enumeration of all sentences in $\mathcal{L}^{*}$. For every $m$-element subset $Y$ of $\left\{c_{n}: n<\omega\right\}$, the complete types $\Gamma(x)$ of $T$ in $\mathcal{L}_{Y}$ are in one-to-one correspondence with the complete types $\Sigma\left(x_{0}, \ldots x_{m-1}, x\right)$ of $T$ in $\mathcal{L}$, and hence there are only countably many of them by the assumption. We let $\left\{\Gamma_{n}(x): n<\omega\right\}$ enumerate all such types $\Gamma(x)$, where $Y$ ranges over all finite subsets of $\left\{c_{n}: n<\omega\right\}$.

By induction on $n<\omega$ we choose an increasing sequence $T_{n}$ of consistent theories with $T_{0}=T$, such that

: (1) each $T_{n}$ contains only finitely many symbols from $\mathcal{L}^{*} \backslash \mathcal{L}$,

: (2) either $\varphi_{n} \in T_{n+1}$ or $\neg \varphi_{n} \in T_{n+1}$,

: (3) if $\varphi_{n} \in T_{n+1}$ and $\varphi_{n}=(\exists x) \psi(x)$, then $\psi(c) \in T_{n+1}$ for some $c \in \mathcal{L}^{*} \backslash \mathcal{L}$, 
: (4) if $\Gamma_{n}(x)$ is consistent with $T_{n}$, then $\Gamma_{n}(c) \subseteq T_{n+1}$ for some $c \in \mathcal{L}^{*} \backslash \mathcal{L}$. The induction is straightforward and we obtain that $T^{*}=\bigcup_{n<\omega} T_{n}$ is a maximal consistent theory in $\mathcal{L}^{*}$. Let $M^{\prime}$ be a countable model of it, therefore by (3), $M^{\prime}=\left(M, a_{n}\right)_{n<\omega}$ where $M=\left\{a_{n}: n<\omega\right\} . M$ is clearly a model of $T$ and (4) guarantees that it is saturated.

Of course, saturation is not a neccessary condition for universality: $\omega+\mathbf{Q}$ is a universal countable linear order but is not a saturated model. We shall not develop this topic further in this article and from now on we shall only consider uncountable models.

\section{Changing the cardinal arithmetic}

When we leave the realm of $G C H$ and its remnants we are more or less left with universes which we construct with forcing and where instances of $G C H$ are violated by the construction of the extension. Theorem 2.7(2) shows that if we are not careful about how we do this, we shall end up basically with no universal models of any sort. Theorem 2.7(3) shows that for certain theories such as linear orders, no matter how careful we are, if we violate $G C H$ sufficiently (including making $2^{\kappa}=2^{\kappa^{+}}$), the universality number at $\kappa^{+}$will jump to the largest possible value of $2^{\kappa^{+}}$. We shall see below that for certain other theories, for example theory of graphs, it is possible to violate $G C H$ as much as we like and still keep the universality number low. This indicates that the ability of having a small universal number in 'reasonable' forcing extensions in which the relevant instances of $G C H$ are violated is a property of the theory itself, which is not possessed by all theories. In fact, in a series of papers, e.g. $[\mathbf{7}],[\mathbf{8}],[\mathbf{6}],[\mathbf{1 5}],[\mathbf{1 6}],[\mathbf{3}]$ the thesis claiming the connection between the complexity of a theory and its amenability to the existence of universal models, has been pursued. In [4] we introduced the following definition, which formalised these notions:

Definition 3.1. We say that a theory $T$ is amenable iff whenever $\lambda$ is an uncountable cardinal satisfying $\lambda^{<\lambda}=\lambda$ and $2^{\lambda}=\lambda^{+}$, while $\theta$ satisfies $\operatorname{cf}(\theta)>$ $\lambda^{+}$, there is a $\lambda^{+}-c c(<\lambda)$-closed forcing notion that forces $2^{\lambda}$ to be $\theta$ and the universality number of $T$ at $\lambda^{+}$to be smaller than $\theta$.

Localising this definition at a particular $\lambda$ we define what is meant by theories that are amenable at $\lambda$.

Connected to this definition there is a somewhat technical definition of high non-amenability (see Definition 0.3 of $[4]$ ). We shall not quote the definition but state only that high non-amenability of $T$ implies that $T$ is not amenable in the sense of Definition 3.1, and that the theory of dense linear order with no endpoints is a prototypical example of a highly non-amenable $T$. We should also comment that the amenability/high non-amenability is envisioned as a dividing in the classification theory of unstable theories. The exact syntactic properties that correspond to this line have not been found yet, but is known that the property $\mathrm{SOP}_{4}$ implies high nonamenability and simplicity implies amenability. We shall not go into these modeltheoretic considerations at this point but refer the reader to the articles mentioned above. Here we shall simply be concerned to give examples and techniques which apply to amenable theories. For simplicity in presentation we shall concentrate on the case of amenability at $\lambda=\aleph_{1}$ but we warn the reader that there are some caveats in looking only at this case-we shall indicate them below. 
3.1. Graphs. The goal of this section is to present a consistency result showing that it is possible to force over a model of $G C H$ to obtain a model with a large continuum and a small universal family of graphs at $\aleph_{1}$. In fact, we shall demonstrate that the theory of graphs is amenable at $\aleph_{1}$, in the sense of Definition 3.1. The earliest theorem to this extent is in Shelah's [13], but unfortunately there is an error, which was noted and corrected by Shelah in [14]. The same year Mekler [9] found a different proof using, unlike [14], a ccc forcing. We shall present Mekler's proof. Let $T$ in this section stand for the model completion of the theory of graphs, so $T$ is a complete model complete theory and we shall show that it is consistent that $C H$ fails and there is a universal model of $T$ of size $\aleph_{1}$.

To start with let $V$ be a model of $G C H$ and let us fix a sequence $\left\langle A_{\alpha}: \alpha<\omega_{1}\right\rangle$ of subsets of $\omega_{1}$ with pairwise intersections countable. Let $\chi$ be a large enough cardinal and we shall work with with elementary substructures of $\langle H(\chi), \in, \ldots)$. The forcing will be an iteration $\left\langle P_{\alpha}, Q_{\beta}: \alpha \leq \omega_{2}, \beta<\omega_{2}\right\rangle$ with finite supports such that each $P_{\alpha}$ is ccc. The first coordinate will have a special role: it will add a generic graph $G^{*}$ on $\omega_{1}$ by finite conditions. Note that $G^{*}$ is a model of $T$. We define the other coordinates by induction on $\alpha$.

For $\alpha \geq 1$, if $P_{\alpha}$ has been defined and is ccc, suppose that a bookkeeping gives us a name $\tau_{\alpha}$, which is a $P_{\alpha}$-name of a model of $T$. We first define $Q_{\alpha}$. In $V$ we fix a continuous sequence $\bar{N}_{\alpha}=\left\langle N_{i}^{\alpha}: i<\omega_{1}\right\rangle$ of countable elementary submodels of $\langle H(\chi), \in, \ldots\rangle$ with $N_{i}^{\alpha} \in N_{i+1}^{\alpha}$ and $\left\langle P_{\beta}, Q_{\beta}: \beta<\alpha\right\rangle, P_{\alpha} \in N_{0}^{\alpha}\left(\right.$ so $\left.\alpha \in N_{0}^{\alpha}\right)$. For $\eta<\omega_{1}$ let $i(\eta)$ denote the first $i$ such that $\eta \in N_{i}^{\alpha}$. Then $Q_{\alpha}$ consists of pairs $q=\left(X_{q}, f_{q}\right)$ such that $X=X_{q}$ is a finite subset of $\omega_{1}$ and $f=f_{q}$ is finite function from $\omega_{1}$ to $A_{\alpha}$ whose range is disjoint from $X$ and which satisfies

$$
\eta \in \operatorname{dom}(f) \Longrightarrow f(\eta) \in N_{i(\eta)+1}^{\alpha} \backslash N_{i(\eta)}^{\alpha} .
$$

The idea is that $Q_{\alpha}$ adds an embedding of $\tau_{\alpha}$ into $G^{*}$. To say this precisely, we define $P_{\alpha+1}$ to consists of elements in $P_{\alpha} * Q_{\alpha}$ such that $p \nmid \alpha$ decides the $\tau_{\alpha}$ structure of $\operatorname{dom}\left(f_{p(\alpha)}\right), \operatorname{ran}\left(f_{p(\alpha)}\right) \subseteq p(0)$ and $f_{p(\alpha)}$ is an embedding of the $\tau_{\alpha}$ structure of $\operatorname{dom}\left(f_{p(\alpha)}\right)$ into $p(0)$.

Easy density arguments show that the $P=P_{\omega_{2}}$ adds an embedding of each $\tau_{\alpha}$ into $G^{*}$ and that if the forcing is indeed ccc, we can do the bookkeeping so to cover all models of $T$ of size $\aleph_{1}$ in the extension. The main point of the proof is to show that the forcing has ccc. The proof uses a certain amalgamation property that is present in the theory of graphs, but not for example in the theory of triangle-free graphs or the theory of linear orders. We shall describe this amalgamation property at the point of the proof where we use it.

We need to pass to a dense set of conditions. Say that for $1 \leq \alpha \leq \omega_{2}, p \in P_{\alpha}$ is complete if for every $\beta<\alpha$ and $i<\omega_{1}$

: (i) $p\left\lceil\beta \cap N_{i}^{\beta}\right.$ is a condition and it determines the $\tau_{\sim}{ }^{\text {-structure of } \operatorname{dom}\left(f_{p(\beta)}\right)}$ and

: (ii) if $r \in N_{i}^{\beta}$ extends $p\left\lceil\beta \cap N_{i}^{\beta}\right.$ and $1 \leq \gamma<\beta$ then $\operatorname{ran}\left(f_{r(\gamma)}\right) \cap p(0) \subseteq$ $\operatorname{ran}\left(f_{p(\gamma)}\right)$.

It can be proved that the set of complete conditions in $P_{\alpha}$ is dense, for all $\alpha$. Both this and the fact that the forcing is ccc are essentially implied by the following Lemma 3.2, which is the main point of the argument.

Let $\mathcal{N}=\left\langle\bar{N}_{\alpha}: \alpha<\omega_{2}\right\rangle$. For the Lemma we need to note that if $p \in P_{\alpha}$ is a complete condition and $N \prec(H(\chi), \in, \mathcal{N}, \ldots)$ countable with $\alpha \in N$, then $p \cap N$ 
is a condition. The proof of this is by induction on $\alpha$, the main case being the case $\alpha=\beta+1$. We have by (i) that $p\left\lceil\beta \cap N_{0}^{\beta}\right.$ determines the $\mathcal{\tau}_{\beta}$ structure of $f_{p(\beta)}$, and we also have that $N_{0}^{\beta} \in N$ so $p \nmid \beta \cap N$, which is a condition by the induction hypothesis is stronger than $p\left\lceil\beta \cap N_{0}^{\beta}\right.$ and hence it too determines the $\mathcal{\sim}_{\beta}$ structure of $f_{p(\beta)}$. We also need to note that the range of $f_{p(\beta)}$ is contained in $N \cap \omega_{1}$, which follows as $f_{p(\beta)}$ is finite.

LEMma 3.2. For any $\alpha \leq \omega_{2}$, if $p \in P_{\alpha}$ is a complete condition, $N \prec(H(\chi), \in$ $, \mathcal{N}, \ldots)$ countable with $\alpha \in N$ and $r \in P_{\alpha} \cap N$ extends $p \cap N$, then $p \cup r$ can be extended to a condition in $P_{\alpha}$ which extends both $p$ and $r^{3}$.

Proof. We shall present the main part of the proof, from which it can be seen where the amalgamation condition is being used. The final part of the proof will only be indicated. Let us assume $\alpha \geq 1$, as otherwise the conclusion is trivial.

We shall need to refer to a following observation about elementary submodels.

Claim 3.3. For any $\gamma \leq \omega_{2}, \gamma \subseteq \bigcup_{i<\omega_{1}} N_{i}^{\gamma}$.

Proof of the Claim. Let $\beta<\gamma$. Since $\gamma \in N_{0}^{\gamma}$ there is $f \in N_{0}^{\gamma}$ which maps $\omega_{1}$ onto $\gamma$. There is $i<\omega_{1}$ such that $f^{-1}(\beta) \in N_{i}^{\gamma}$ and then $\beta \in N_{i}^{\gamma} \cdot \star_{3.3}$

The construction of the desired condition is by induction on $k<\omega$, where the induction will stop after some finite number of stages. At each stage we define

$$
N_{k} \prec(H(\chi), \in, \ldots) \text { countable, } r_{k} \in P_{\alpha}, a_{k} \subseteq \operatorname{dom}(p) \backslash\{0\},\left(s_{\beta}^{k}\right)_{\beta \in a_{k}}, \gamma_{k}
$$

where each $s_{\beta}^{k}$ is a finite graph on a subset of $\omega_{1}$. For each $k>0, \gamma_{k}$ is a special element of $a_{k}$, called the leading ordinal, with $\gamma_{0}=\infty$. The elements of $a_{k}$ are called the active ordinals. We denote $\delta_{k}=N_{k} \cap \omega_{1}$. The following are our inductive hypotheses:

- If $\beta \in a_{k}$ then $s_{\beta}^{k}$ is a subgraph of $r_{k}(0) \cup \operatorname{ran}\left(f_{p(\beta)}\right)$ (which in itself is a graph), and the universe of $s_{\beta}^{k} \cap r_{k}(0)$ is exactly $s_{\beta}^{k} \cap \delta_{k}$,

- if $k>0$ then there is $i<\omega_{1}$ such that $N_{k}=N_{i}^{\gamma_{k}}$,

- $r_{k} \in N_{k} \cap P_{\gamma_{k}}$ and it extends $p\left\lceil\gamma_{k} \cap N_{k}\right.$ and $r_{m}\left\lceil\gamma_{k}\right.$ for all $m<k$,

- if $\beta \leq \gamma_{k}$ is active, then $r_{k} \uparrow \beta$ determines the $\mathcal{\tau}_{\beta}$ structure of $\operatorname{dom}\left(f_{p(\beta)}\right) \cup$ $\bigcup_{m \leq k} \operatorname{dom}\left(f_{r_{m}(\beta)}\right) \cap \delta_{k}$ and $r_{k} \uparrow \beta$ forces that $f_{p(\beta)} \cup \bigcup_{m \leq k} f_{r_{m}(\beta)} \uparrow \delta_{k}$ is an isomorphism of this structure with $s_{\beta}^{k}$,

- if $\beta^{\prime} \neq \beta^{\prime \prime}$ are both in $a_{k}$ and satisfy $A_{\beta^{\prime}} \cap A_{\beta^{\prime \prime}} \nsubseteq \delta_{k}$, then $s_{\beta^{\prime}}^{k} \cap s_{\beta^{\prime \prime}}^{k} \subseteq p(0)$.

We say that for $\beta \in a_{k}$, the elements of $\operatorname{dom}\left(f_{p(\beta)}\right)$ that have been used at the stage $k$ are those in $\operatorname{dom}\left(f_{p(\beta)}\right) \cap \delta_{m}$, where $m=\max \left\{l \leq k: \gamma_{l} \geq \beta\right\}$ (since $\gamma_{0}=\infty$, this maximum is well defined). We denote the set of such ordinals by $u_{\beta}^{k}$.

The induction is not difficult to do: we let $N_{0}=N, r_{0}=r$ and $a_{0}=\operatorname{dom}(p) \backslash$ $\{0\} \cap N$. For $\beta \in a_{0}$ let $s_{\beta}^{0}=\operatorname{ran}(r(\beta))$ with the structure induced by $r(0)$ - this is well defined as $p \cap N \leq r$. If we have defined all the relevant objects at the stage $k$, do the following if possible: choose a minimal $\gamma=\gamma_{k+1} \in a_{k}$ such that there is an unused element of $\operatorname{dom}\left(f_{p(\gamma)}\right)$. Let $i$ be the minimal such that there is an element of $\operatorname{dom}\left(f_{p\left(\gamma_{k+1}\right.}\right) \backslash u_{\gamma_{k+1}}^{k}$ in $N_{i}^{\gamma}$ and satisfying $N_{k} \cap \gamma_{k+1} \subseteq N_{i}^{\gamma_{k+1}}$ (note that such $i$ exists by Claim 3.3). Let $N_{k+1}=N_{i}^{\gamma_{k+1}}$ and $a_{k+1}=a_{k} \cup\left[\operatorname{dom}(p) \cap N_{k+1} \cap \gamma_{k+1}\right]$. If

\footnotetext{
${ }^{3}$ By $p \cup r$ we mean the function $q$ whose domain is $\operatorname{dom}(p) \cup \operatorname{dom}(r)$, with $q(0)=p(0) \cup r(0)$ and $q(\beta)=\left(X_{p(\beta)} \cup X_{r(\beta)}, f_{p(\beta)} \cup f_{r(\beta)}\right)$ for $\beta>0$ in $\operatorname{dom}(q)$.
} 
no such choice is possible, stop the induction. Note that $m \leq k \Longrightarrow \gamma_{m} \leq \gamma_{k}$ and that the induction must be stopped at some stage $k^{*}$ as all ordinals that appear as unused at any stage come from the finite set $\bigcup_{\gamma \in \operatorname{dom}(p) \backslash\{0\}} \operatorname{dom}\left(f_{p(\gamma)}\right)$ (and once an ordinal is used it remains so). Let $\gamma^{*}=\gamma_{k^{*}}$.

We shall now proceed to define a condition $q$ in $N_{\gamma^{*}} \cap \gamma^{*}$. We shall need the following claim.

Claim 3.4. For all $k \leq k^{*}, \bigcup_{m \leq k} N_{m} \cap \gamma_{k} \subseteq N_{k}$.

Proof of the Claim. The proof is by induction on $k$. If $k=0$, the situation is clear. For the case of $k+1$, we have by the induction hypothesis that for $m \leq k$, $N_{m} \cap \gamma_{k} \subseteq N_{k}$. Since $\gamma_{k+1} \leq \gamma_{k}$, certainly $N_{m} \cap \gamma_{k+1} \subseteq N_{k}$ and hence it suffices to show that $N_{k} \cap \gamma_{k+1} \subseteq N_{k+1}$. This conclusion follows by the choice of $N_{k+1}$ • $\star_{3.4}$

Suppose that $k \leq k^{*}$ and $m \leq k$. Then $r_{m} \in N_{m}$ so all the finitely many ordinals involved in the construction of $r_{m} \uparrow \gamma_{k}$ are in $N_{m} \cap \gamma_{k}$, and hence in $N_{k}$ by Claim 3.4. Therefore $r_{m} \uparrow \gamma_{k} \in N_{k}$.

At this point of the proof we shall get to use the Amalgamation Condition $(*)$, which we define in terms of a partially ordered class $(K, \leq)$ of structures:

$(K, \leq)$ satisfies a.c. (*) if for any elements $A, B,\left\{C_{i}: i \in I\right\}$ of $K$ satisfying

- any two of these structures agree on their intersection

- whenever $i \neq j$ then either $C_{i} \cap C_{j} \subseteq A$ or $C_{i} \cap C_{j} \subseteq B$, there is a structure in $K$ which extends all $A, B,\left\{C_{i}: i \in I\right\}$.

Obviously, the class of graphs with the induced subgraph relation satisfies the a.c. $(*)$ since we can simply take a union of all the graphs involved. ${ }^{4}$

Suppose now that $\beta^{\prime} \neq \beta^{\prime \prime} \in a_{k^{*}}$. By the induction hypothesis either $A_{\beta^{\prime}} \cap$ $A_{\beta^{\prime \prime}} \nsubseteq \delta_{k^{*}}$, in which case $s_{\beta^{\prime}}^{k^{*}} \cap s_{\beta^{\prime \prime}}^{k^{*}} \subseteq p(0)$ or $A_{\beta^{\prime}} \cap A_{\beta^{\prime \prime}} \subseteq \delta_{k^{*}}$ and so $s_{\beta^{\prime}}^{k^{*}} \cap s_{\beta^{\prime \prime}}^{k^{*}} \subseteq$ $r_{k^{*}}(0)$. This says that, according to the a.c. $(*)$ property, we can define a graph that extends $p(0) \cap \delta_{k^{*}} \cup r_{k^{*}}(0) \cup \bigcup_{\beta \in a_{k^{*}}} s_{\beta}^{k^{*}} \cap \delta_{k^{*}}$. Let $s$ be such a graph. We define $q^{\prime}$, a candidate for a condition in $P_{\alpha}$. Let $q^{\prime}(0)=s$ and for $\beta \in \bigcup_{k \leq k^{*}} \operatorname{dom}\left(r_{k}\right) \cup a_{k^{*}}$ let $q^{\prime}(\beta)=p(\beta) \cap \delta_{k^{*}} \cup \bigcup_{k \leq k^{*}} r_{k}(\beta)$. Note that $q^{\prime}$ as a function is an element of $N_{k^{*}}$. We now claim that $q^{\prime}$ is a condition in $P_{\alpha}$. By induction on $1 \leq \beta \leq \alpha$ we prove that $q^{\prime}\left\lceil\beta \in P_{\beta}\right.$. For $\beta=1$ this follows from the choice of $s$ and for $\beta$ limit this is clear. Suppose that $\beta=\gamma+1$ and $\gamma$ is in $\operatorname{dom}\left(q^{\prime}\right)$. If $\gamma \notin \operatorname{dom}(p)$ then $\gamma \in \bigcup_{k \leq k^{*}} \operatorname{dom}\left(r_{k}\right)$ and $q^{\prime}(\gamma)=\bigcup_{k \leq k^{*}} r_{k}(\gamma)$. This is well defined by the choice of $r_{k}$ 's. If $\gamma \in \operatorname{dom}(p) \backslash \bigcup_{k \leq k^{*}} \operatorname{dom}\left(r_{k}\right)$, then $q^{\prime}(\gamma)=p(\gamma) \cap \delta_{k^{*}}$, so $q^{\prime} \uparrow \beta$ is a condition because $p$ satisfies (i) in the definition of completeness. Finally it may happen that $\gamma \in \operatorname{dom}(p) \cap \bigcup_{k \leq k^{*}} \operatorname{dom}\left(r_{k}\right)$. In particular we have $\beta \in a_{k^{*}}$. Let $k$ be maximal such that $\gamma \leq \gamma_{k}$. We claim that $\operatorname{dom}\left(f_{p(\gamma)}\right) \cap \delta_{k^{*}}=\operatorname{dom}\left(f_{p(\gamma)}\right) \cap \delta_{m}$. If this were not to be the case then at the stage $k$ of the induction there would be an unused ordinal in $\operatorname{dom}\left(f_{p(\gamma)}\right)$, so $\gamma=\gamma_{k+1}$, a contradiction. Having established the existence of $q^{\prime}$ we now need to keep going to extend to a condition which extends each $r_{k}$. This is done in stages using the assumptions of completeness, for which we refer the reader to the original article.

We now indicate how Lemma 3.2 implies that the forcing is ccc. We assume that we have proved that the set of complete conditions is dense, so we only work

\footnotetext{
a.c. $(*)$.

${ }^{4}$ In $[\mathbf{9}]$, Lemma 1 , it is shown that a better known $\mathcal{P}\left(3^{-}\right)$amalgamation property implies
} 
with them. So assume that $\mathcal{A}$ is a maximal antichain of complete conditions and let $N$ be a rich enough countable elementary submodel with $\mathcal{A} \in N$. We claim $\mathcal{A}=\mathcal{A} \cap N$, so $\mathcal{A}$ is countable. For this we have to show that $\mathcal{A} \cap N$ is a maximal antichain. Let $p$ be any complete condition. By elementarity there is $r \in N \cap \mathcal{A}$ which extends $p \cap N$. By Lemma 3.2 there is a common extension of $p$ and $r$. This proves the statement we claimed.

We remark that the above result, as it involves ccc forcing iteration, can be easily generalised to cardinals of the form $\lambda^{+}$for some $\lambda$ satisfying $\lambda^{<\lambda}=\lambda$, as Mekler does in Theorem 8 of [9]. Therefore the theory of random graphs is amenable in the sense of Definition 3.1.

3.2. Triangle-free Graphs. Having established that the theory of graphs is amenable the next natural question to ask to what extent the amalgamation condition $(*)$ was necessary for this conclusion. A very good example to consider is that of the model completion of the theory of triangle-free graphs. In the sense of model-theoretic complexity, this theory provides a prototypical example of a non-simple 'simple enough' theory, but also because this theory fails the a.c $(*)$. Namely

EXAMPLE 3.5. Let $C_{l}$ be a graph consisting of the edge $\left(c_{l}, c_{l+1 / \bmod 3}\right)$, for $l<3$, let $A=C_{0}$ and $B=C_{1}$. These structures satisfy the premises of the condition a.c (*) but any graph extending $C_{0}, C_{1}$ and $C_{2}$ contains the triangle $\left\{c_{0}, c_{1}, c_{2}\right\}$.

Therefore methods of [9] do not apply to triangle-free graphs. Džamonja and Shelah proved in [3] that the theory of the model completion of triangle-free graphs is nevertheless amenable. We explain the main ideas of this proof.

Let us first concentrate on $\aleph_{1}$. In the final extension we shall have $2^{\aleph_{0}}=2^{\aleph_{1}}=$ $\aleph_{3}$ and the universality number of the class of the triangle free graphs of size $\aleph_{1}$ will be $\aleph_{2}$. The values $\aleph_{2}$ and $\aleph_{3}$ are rather flexible, but we concentrate on these for concreteness. We consider the class $K$ all finite triangle-free graphs $G$ whose universe is a subset of $\omega_{1}$ and that have the property that for every non-zero limit $\delta<\omega_{1}, G\left\lceil\delta\right.$ is a 'reflection' of $G$ in the sense that for every pair $a_{0}, a_{1} \in G\lceil\delta$, if there is $c$ with $a_{l} R c$ for $l<2$, then there is such $c$ in $G \uparrow \delta$. We can order this class by the relation of being an induced subgraph. Let us call this partially ordered class the approximation family. A petition on the approximation family is a directed subset of it of size $\aleph_{1}$. Notice that if we are given a petition $\Gamma$ then its union is a graph of size $\aleph_{1}$, and by directedness, this graph is triangle-free. We call this graph $M_{\Gamma}$. Notice that by reenumerating, every triangle free graph of size $\aleph_{1}$ is isomorphic to one whose family of finite subgraphs forms a petition on the approximation family. We shall be interested in quorumed petitions, which are those petitions that contain an isomorphic copy of every finite triangle-free graph on $\omega_{1}$ through an isomorphism which moves every ordinal $\alpha$ to an ordinal $\beta$ of finite distance with $\alpha$. The existence of such quorumed petitions is not something we prove in $Z F C$, but we force them.

Let us now describe the forcing. We start with a model of $G C H$ an in it we denote by $\mathcal{T}$ the tree ${ }^{\omega_{1}>} \omega_{1}$. We first add $\aleph_{3}$ many Cohen subsets of $\omega_{1}$ by countable conditions. Notice that this introduces $\aleph_{3}$ many branches to $\mathcal{T}$. Call the resulting universe $V$. We follow this with an iteration $\left\langle P_{\alpha}, Q_{\alpha}: \alpha<\omega_{2}\right.$ in which each step $Q_{\alpha}$ is itself an iteration of $\omega_{3}$ steps, of ccc forcing, which we shall call $a$ block. In eãch block we shall have a preliminary forcing and an iteration of 
length $\omega_{3}$. We first describe the iteration. It will have two kinds of coordinates. In each coordinate of the first kind. of it we are given a name for a petition $\underset{\sim}{\Gamma}$ on the approximation family and we force to embed $M_{\Gamma}$ into $M_{\Gamma^{*}}$ for some quorumed petition $\Gamma_{\sim}^{*}$. By remarks above, for our final universality result it will be sufficient to ensure that there are $\aleph_{2}$ triangle-free graphs on $\omega_{1}$ which embed all $M_{\Gamma^{*}}$ for quorumed petitions $\Gamma^{*}$. By bookkeeping, we shall at each stage $\alpha<\omega_{2}$ of the main iteration assure by $Q_{\alpha}$ of forcing that we have dealt with all quorumed petitions in $V^{P_{\alpha}}$. In fact we shall assure that there is in $V^{P_{\alpha+1}}$ a single triangle-free graph $G_{\alpha}^{*}$ on $\omega_{1}$ which embeds all $M_{\Gamma^{*}}$ for quorumed petitions $\Gamma^{*}$ in $V^{P_{\alpha}}$. This is where the preliminary forcing of the block $\alpha$ comes in: in it we introduce a system $S_{\alpha}$ of members of the approximation family indexed by the nodes in $\mathcal{T}$ in a such a way that each branch through $\mathcal{T}$ gives a petition in the approximation family. It is easy to see that the union of this system is a triangle-free graph on $\omega_{1}$, which will be our $G_{\alpha}^{*}$. In the second kind of coordinates in the block $\alpha$ we shall be embedding a quorumed petition $\underset{\sim}{H}$ given by the bookkeeping into the subsystem of $S_{\alpha}$ given by the elements indexed by the nodes on some branch of $\mathcal{T}$. This assures that $M_{H}$ embeds into $G_{\alpha}^{*}$.

The main point of the proof is to make sure that each individual forcing in a block is ccc, and in assuring so in the second kind of coordinates we get to use an amalgamation property possessed by the elements of the approximation family $K$ :

Suppose that $M_{0}, N_{0}, M_{1}, M_{2}, N_{1}, N_{2}$ and $M$ are in $K$ such that

- $M_{0}=M_{1} \cap M_{2}$ and $M_{1}$ is isomorphic with $M_{2}$ by an isomorphism which is identity on $M_{0}$,

- $N_{0}=N_{1} \cap N_{2}$ and $N_{1}$ is isomorphic with $N_{2}$ by an isomorphism which is identity on $N_{0}$,

- each $M_{i}$ is an induced subgraph of the corresponding $N_{i}$ and the universe of $M_{l}$ consists of even ordinals in the universe of $N_{l}$,

- there are limit ordinals $\delta_{0}<\delta_{1}<\delta_{2}$ such that $N_{l} \subseteq \delta_{l}$ for each $l<3$,

- the universe of $M$ is contained in the even ordinals and $M_{1}, M_{2}$ are induced subgraphs of $M$.

Then there is $N \in K$ whose induced subgraphs include $M, N_{1}$ and $N_{2}$.

This property is called workability in $[\mathbf{3}]$. Notice that checking that it is true really uses the definition of the approximation family, not only the properties of the class of triangle-free graphs.

The proof in the case $\lambda^{+}$for $\lambda=\lambda^{<\lambda}$ in place of $\aleph_{1}$ has to deal with a strong version of $\lambda^{+}$-cc needed in order to iterate, which introduces additional complications which we shall not describe here. The structure of the proof as described above uses a tree of models rather than a linearly organised structure like in [9]. The price we have to pay is that the final universe does not have one universal model, rather just a small universal family. The following question is still open:

QUESTION 3.6. Is it consistent to have a universal triangle-free graph on $\aleph_{1}$ and not $\mathrm{CH}$ ?

3.3. Linear orders. The next step to consider in our increasing level of complexity of theories is a theory that does not have a workable approximation family. Such a theory is the theory of dense linear orders. Namely, the main result of Kojman-Shelah [7] is that this theory is highly non-amenable, and the proof we presented for triangle-free graphs cannot be adopted to this case- as it would show 
that the theory is amenable. As an exercise, the reader may check that natural definitions of approximation families for this class will fail to be workable. However, in [12] Shelah proved that it is consistent that there is a universal linear order of size $\aleph_{1}$ in a model where $C H$ fails ${ }^{5}$. In the terminology of the rest of this paper, this shows that the theory of dense linear orders is amenable at $\aleph_{1}$. Note that the method of the proof uses oracle-proper forcing, which is a technique limited to $\aleph_{1}$. Again by high non-amenability we conclude that the analogue of [12] cannot be obtained at cardinals larger than $\aleph_{1}$, and at the same time that amenability is not implied by amenability at $\aleph_{1}$.

3.4. Amenability at $\aleph_{1}$. Our presentation so far leaves open the question if every 'reasonable' theory $T$ is amenable at $\aleph_{1}$. Namely, let $D(T)$ denote the set of complete types over the empty set in finitely many variables. It is known that if this set is uncountable then it has to have the cardinality of the continuum, and it is easy to see that every type in $D(T)$ must be realised in the universal model. Therefore if $D(T)$ is uncountable there cannot be a universal model of $T$ in $\aleph_{1}$ if $\mathrm{CH}$ fails. It remains to ask what happens if $D(T)$ is countable, namely if it is possible that every $T$ with $D(T)$ countable is amenable at $\aleph_{1}$. A negative answer to this is given in $\S 1$ of [7], where there is an example of a theory with countable $D(T)$ which has a universal model at $\aleph_{1}$ iff $C H$ holds.

Conclusion. In conclusion, we have presented the methods that are currently available for making the universality number at $\aleph_{1}$ small while failing $C H$. These methods come with amalgamation-type requirements on the theory in question and we have discussed the prototypical examples of theories that satisfy or not these amalgamation properties. We have discussed the division amenability/high nonamenability defined in terms of the ability of a theory to have a small universality number in circumstances where the relevant instances of $G C H$ are violated. We end by mentioning that there is a programme of characterising this division in syntactic terms i.e. in terms that do not discuss models of a theory but properties of its types and formulae. The present state of this programme is described in the Introduction to $[4]$.

\section{References}

[1] C. Chang and H.J. Keisler, Model Theory (3. edition), Studies in Logic, vol. 73, NorthHolland, (Amsterdam-New York-Oxford-Tokyo), 650 pp.,(1990).

[2] M. Džamonja, Club guessing and the universal models, Notre Dame Journal for Formal Logic, vol. 46, No. 3, pp. 283-300, (2005).

[3] M. Džamonja and S. Shelah, On the existence of universals, Archive for Mathematical Logic, vol. 43, pp. 901-936, (2004).

[4] M. Džamonja and S. Shelah, On properties of theories which preclude the existence of universal models, Annals of Pure and Applied Logic, vol. 139, no. 1-3, pp. 280-302, (2006).

[5] F. Hausdorff, Grundzüge einer Theorie der geordneten Mengenlehre, Mathematische Annalen, vol. 65, pp. 435-505. (In German) (1908).

[6] M. Kojman, Representing embeddability as set inclusion, Journal of the London Mathematical Society (2nd series), vol 58, no. 185, Part 2, pp. 257-270 (1998).

[7] M. Kojman and S. Shelah, Non-existence of Universal Orders in Many Cardinals, Journal of Symbolic Logic, vol. 57, pp. 875-891, (1992).

\footnotetext{
${ }^{5}$ Unfortunately, the published proof of this very important result is very sketchy and the author of this article would not claim that she understands it completely. Shelah has kindly explained some of the major missing and inaccurate points in a recent conversation and promised to make a written version available.
} 
[8] M. Kojman and S. Shelah, The universality spectrum of stable unsuperstable theories, Annals of Pure and Applied Logic, vol. 58, pp. 57-72, (1992).

[9] A. H. Mekler, Universal structures in power $\aleph_{1}$, Journal of Symbolic Logic vol. 55, no.2, pp. 466-477, (1990).

[10] M. Morley and R. Vaught, Homogeneous universal models, Math. Scand., vol. 11, pp. 37-57, (1962).

[11] S. Shelah, Classification Theory, revised ed., Studies in Logic, vol. 73, North-Holland (Amsterdam-New York-Oxford-Tokyo), 705 pp., (1990).

[12] S. Shelah, Independence results, Journal of Symbolic Logic, vol. 45, pp. 563-573, (1980).

[13] S. Shelah, On universal graphs without instances of $C H$, Annals of Pure and Applied Logic, vol. 26, pp. 75-87, (1984).

[14] S. Shelah, Universal graphs without instances of GCH: revisited, Israel Journal of Mathematics, vol. 70, no. 1, pp. 69-81, (1990).

[15] S. Shelah, The Universality Spectrum: Consistency for more classes in Combinatorics, Paul Erdös is Eighty, Bolyai Society Mathematical Studies vol. 1, pp. 403-420, (1993). (proceedings of the Meeting in honor of P. Erdös, Keszthely, Hungary 7. 1993, an improved version available at http://www.math.rutgers.edu/ shelarch).

[16] S. Shelah, Toward classifying unstable theories, Annals of Pure and Applied Logic vol. 80, pp. 229-255, (1996).

[17] R. Vaught, Denumerable models of complete theories, in lnfinitistic Methods (Pergamon, London) pp. 303-321, (1961).

School of Mathematics, University of East Anglia, Norwich NR4 7TJ, UK

E-mail address: h020@uea.ac.uk 